ARTICLE

Received 1 Apr 2013 | Accepted 17 Jun 2013 | Published 16 Jul 2013

DOl: $10.1038 /$ ncomms3163 OPEN

\title{
Structural modulation of gut microbiota in life-long calorie-restricted mice
}

Chenhong Zhang ${ }^{1}$, Shoufeng $\mathrm{Li}^{2}$, Liu Yang ${ }^{2}$, Ping Huang ${ }^{2}$, Wenjun $\mathrm{Li}^{2}$, Shengyue Wang ${ }^{3}$, Guoping Zhao ${ }^{3}$, Menghui Zhang ${ }^{1}$, Xiaoyan Pang ${ }^{1}$, Zhen Yan ${ }^{4,5}$, Yong Liu² \& Liping Zhao ${ }^{1,6}$

Calorie restriction has been regarded as the only experimental regimen that can effectively lengthen lifespan in various animal models, but the actual mechanism remains controversial. The gut microbiota has been shown to have a pivotal role in host health, and its structure is mostly shaped by diet. Here we show that life-long calorie restriction on both high-fat or lowfat diet, but not voluntary exercise, significantly changes the overall structure of the gut microbiota of C57BL/6 J mice. Calorie restriction enriches phylotypes positively correlated with lifespan, for example, the genus Lactobacillus on low-fat diet, and reduces phylotypes negatively correlated with lifespan. These calorie restriction-induced changes in the gut microbiota are concomitant with significantly reduced serum levels of lipopolysaccharidebinding protein, suggesting that animals under calorie restriction can establish a structurally balanced architecture of gut microbiota that may exert a health benefit to the host via reduction of antigen load from the gut.

\footnotetext{
${ }^{1}$ State Key Laboratory of Microbial Metabolism, School of Life Sciences and Biotechnology, Shanghai Jiao Tong University, Shanghai 200240, China.

${ }^{2}$ Key Laboratory of Nutrition and Metabolism, Institute for Nutritional Sciences, Shanghai Institutes for Biological Sciences; Graduate School of the Chinese Academy of Sciences; Chinese Academy of Sciences, Shanghai 200031, China. ${ }^{3}$ Shanghai-MOST Key Laboratory for Disease and Health Genomics, Chinese National Human Genome Centre, Shanghai 201203, China. ${ }^{4}$ Departments of Medicine-Cardiovascular Medicine and Pharmacology, University of Virginia, Charlottesville, Virginia 22908, USA. ${ }^{5}$ Center for Skeletal Muscle Research, Robert M. Berne Cardiovascular Research Centre, University of Virginia, Charlottesville, Virginia 22908, USA. ${ }^{6}$ Ministry of Education Key Laboratory of Systems Biomedicine, Shanghai Centre for Systems Biomedicine, Shanghai 200240, China. Correspondence and requests for materials should be addressed to L.Z. (email: Ipzhao@sjtu.edu.cn) or to Y.L. (email: liuy@sibs.ac.cn).
} 
S ince the initial study demonstrating the health-promoting and lifespan-extending effects of calorie restriction (CR) in mice $^{1}$, benefits related to alleviating the metabolic syndrome have been observed in many mammals, including non-human primates and humans ${ }^{2,3}$. Despite various efforts, the actual mechanism remains controversial ${ }^{4,5}$.

Two recent, life-long metabonomic studies in dogs and monkeys revealed that CR was associated with changes in urinary bacterial metabolites, suggesting a potential connection among the gut microbiota, CR and aging ${ }^{6,7}$. Humans are considered supraorganisms with a vastly diverse and highly populated microbiota in the gut ${ }^{8,9}$, which can function as a metabolic organ to significantly modulate nutrition, metabolism and the immunity of its host ${ }^{10}$. After the host digests and absorbs nutrients from the diet, the remaining part will reach the colon to maintain a highly diverse and populated chemostatic culture ${ }^{11,12}$. The composition and amount of the diet work as a dominant force in shaping the gut microbiota ${ }^{12,13}$.

Changes in the gut microbiota responding to different diets, such as a high-fat diet versus normal chow, may have a pivotal role in the development of obesity and related diseases ${ }^{13-}$ 18. Gut microbiota disrupted by a high-fat diet may produce higher amounts of endotoxin and increase gut permeability, leading to a higher plasma level of endotoxin, a higher level of inflammation and eventually the development of metabolic disorders ${ }^{13,14,19,20}$. Our recent study showed that one endotoxin-producing strain isolated from the gut of an obese human caused obesity and insulin resistance in germ-free mice. These bacterium-induced obese mice had a significantly elevated serum endotoxin load and increased systemic and local inflammation, indicating a causative role of the endotoxinproducing members in the gut microbiota in metabolic syndrome ${ }^{21}$. However, it remains to be elucidated how far and to what direction the gut microbiota can be shifted by changing only the amount of food intake such as in CR treatment.

Recently, Zhou et al. ${ }^{22}$ identified genetic modulators of aging by analysing mid-life gene expression in the liver of a mouse model with life-long dietary and exercise interventions. In that study, male C57BL/6 J mice were subjected to either a low-fat diet (10\% fat, D12450B, Research Diets) or a high-fat diet (60\% fat, D12492, Research Diets). For each type of diet, animals were divided into three groups: (1) fed ad libitum with sedentary activity in the cage (LFD or HFD), (2) fed $70 \%$ of the ad libitum (LFD + CR or HFD + CR) or (3) fed ad libitum with voluntary wheel-running exercise (LFD + Ex or HFD + Ex). Each group had 30 individually caged animals, and the entire trial lasted almost 4 years until all animals died ${ }^{22}$. The longest living and healthiest animals were in the LFD + CR group. Relative to the LFD group, their median lifespan (153 weeks) and maximum lifespan ( $185.5 \pm 1.6$ weeks) increased by approximately $20 \%$ and $25 \%$, respectively. The LFD + CR group also exhibited the lowest and most stable body weight and fat content, as well as the best metabolic phenotypes, such as glucose homoeostasis and serum lipid profile, at the different indicated ages throughout their lifespan. The HFD group had the shortest lifespan and the worst metabolic phenotypes. Compared with the HFD control group, restricted high-fat diet intake $(\mathrm{HFD}+\mathrm{CR})$ resulted in dramatic extensions of the median and maximum lifespans (both by $\sim 36 \%$ from 101 to 137 weeks and from $118.8 \pm 1.5$ to $161.9 \pm 1.5$ weeks, respectively), which became similar to those of the LFD (127 and $148.7 \pm 3.1$ weeks, respectively) and LFD + Ex (131 and $159.6 \pm 3.7$ weeks, respectively) groups. In addition, similar metabolic phenotypes were observed among these groups. Voluntary running exercise resulted in a significant increase in the median and maximum lifespan (by $\sim 13 \%$, from 101 to 114 weeks, and by $\sim 18 \%$, from $118.8 \pm 1.5$ to $139.7 \pm 1.9$ weeks, respectively) when animals were fed ad libitum on high-fat diet but not on low-fat diet. Together, these data demonstrate that obesity-related metabolic syndrome is highly associated with accelerated aging and reduced lifespan. CR can more effectively alleviate diet-associated metabolic disorders and attenuate aging than voluntary exercise, leading to a prolonged healthy lifespan, consistent with early studies in rats ${ }^{22}$.

In the current study, we use faecal and serum samples from the same animal trial as Zhou et al. ${ }^{22}$ to investigate the impact of lifelong CR and voluntary exercise on the endotoxin load and architecture of gut microbiota and pinpoint the association between a specific combination of populations in the gut microbiota and the variations in healthy phenotype and lifespan of their hosts. Our findings suggest that an improved architecture of gut microbiota may be a critical element in mediating the health-promoting actions of $\mathrm{CR}$, highlighting the potential of modulation for gut microbiota in developing effective anti-aging dietary interventions.

\section{Results}

Overall structural changes of gut microbiota in life-long CR. To determine whether the CR-mediated protection of mice against obesity-associated metabolic syndrome and promotion of healthy aging are associated with alteration of gut microbiota structure, we first profiled the overall structural changes of gut microbiota from all available animals at 62,83 and 141 weeks of age by bar-coded pyrosequencing of the V3 region of $16 \mathrm{~S}$ rRNA genes. Of 293,557 valid reads from 288 samples with an average of 1,019 reads per sample ( \pm 205 s.d.), 4,613 species-level operational taxonomic units (OTUs) were delineated using $97 \%$ as a homology cut-off value (Supplementary Fig. S1).

$\beta$-Diversity analysis can indicate the extent of similarity between microbial communities by measuring the degree to which membership or structure is shared between communities ${ }^{23}$. Based on the data matrix of the weighted UniFrac distance, unweighted pair-group method using arithmetic averages and principal coordinate analysis showed both age-dependent and diet-responsive structural rearrangement of gut microbiota (Fig. 1 and Supplementary Fig. S2). Although no significant age-related shift of gut microbiota was observed around mid-life ages (between 62 and 83 weeks), the gut microbiota from all groups of mice alive at the late-life age of 141 weeks displayed the same trend of moving into an 'aging space.' Conversely, separated microbiota clusters were observed in high-fat diet-fed mice relative to low-fat diet-fed mice at the two mid-life ages. Moreover, in parallel with its profound effects on health improvement and longevity, CR showed more prominent impact on the overall architecture of gut microbiota than exercise, particularly with unique microbiota clusters detected in the LFD + CR group both at mid-life and late-life ages. The differences between the gut microbiota of animals with or without voluntary exercise were not significant in the present study. These results suggest a possible correlation of the clustering pattern of gut microbiota with the health conditions in response to life-long nutritional intervention.

Specific phylotypes modulated by life-long CR. As an algorithm to robustly identify features that are statistically different among biological classes, linear discriminant analysis (LDA) effect size $(\text { LEfSe })^{24}$ was employed to identify specific phylotypes responding to life-long CR at both mid-life (62 weeks of age) and late life (141 weeks of age). We did not analyse data at 83 weeks of age because there is no significant age-related shift of gut microbiota between 62 and 83 weeks. 

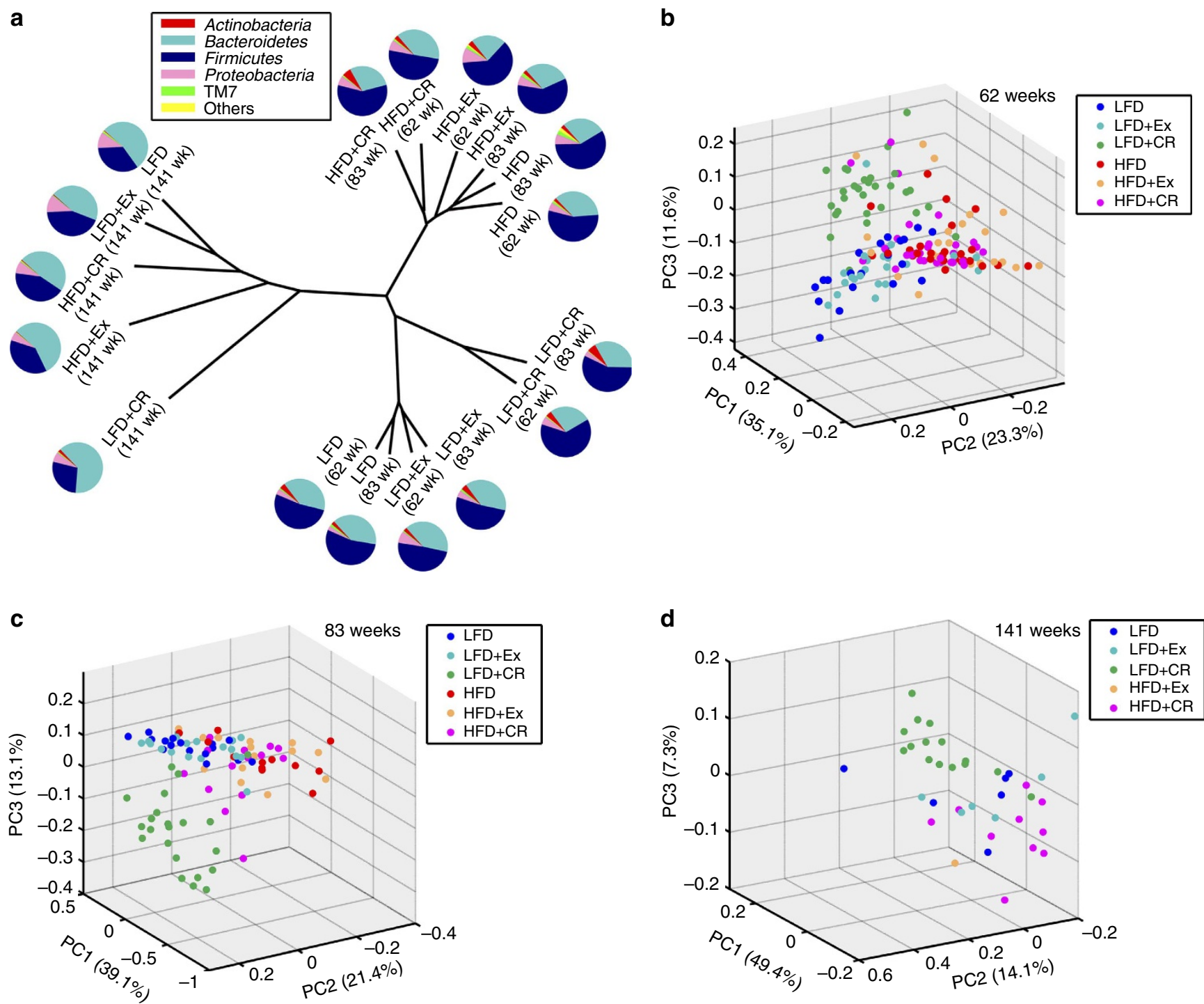

Figure 1 | Age-dependent and diet-responsive alteration trajectories of global gut microbiota structures. (a) Unweighted pair-group method using arithmetic average based on the weighted UniFrac distance of gut microbiota from the six groups of mice at 62,83 and 141 weeks (wk) of age. The average relative abundance (\% of total $16 \mathrm{~S}$ rRNA gene $\mathrm{V} 3$ region sequences) of bacterial lineages of the gut microbiota within each group of mice is displayed as pie charts at the phylum level. Weighted UniFrac principal coordinate analysis of animals at (b) 62 (LFD, $n=21 ; L F D+C R, n=29 ; L F D+E x$, $n=22 ; \mathrm{HFD}, n=28 ; \mathrm{HFD}+\mathrm{CR}, n=29 ;$ and HFD + Ex, $n=23)$, (c) 83 (LFD, $n=16 ; \mathrm{LFD}+\mathrm{CR}, n=22 ; \mathrm{LFD}+\mathrm{Ex}, n=19 ; \mathrm{HFD}, n=12 ; \mathrm{HFD}+\mathrm{CR}, n=14 ;$ and HFD + Ex, $n=15)$ and (d) 141 (LFD, $n=6$; LFD + CR, $n=15 ;$ LFD + Ex, $n=6 ;$ HFD, $n=0 ;$ HFD + CR, $n=10$; and HFD + Ex, $n=1)$ weeks of age.

In mid-life, 34 phylotypes at the OTU level were discovered as high-dimensional biomarkers for separating gut microbiota between LFD and LFD + CR mice (Fig. 2a and Supplementary Table S1). Sixteen of these OTUs were higher, and eighteen were lower in the CR than in the ad libitum group. For example, the abundances of these selected phylotypes in Streptococcaceae (OTU65 belonging to Lactococcus) and TM7 (OTU98) were lower in CR animals. Interestingly, OTU45 in the genus Lactobacillus was one of the most predominant phylotypes in bacterial communities of LFD + CR mice but was notably low in LFD mice (12.4\% versus $0.05 \%$, respectively; $P<0.001$, one-way ANOVA).

At the late-life age of 141 weeks, 27 OTUs were higher and 27 were lower in the LFD + CR group than in the LFD group (Fig. $2 \mathrm{~b}$ and Supplementary Table S2). Ten of these OTUs were also significantly different between the two treatment groups in mid-life. For example, although OTU45 in the genus Lactobacillus was not the predominant phylotype in bacterial communities of LFD + CR mice, the relative abundance of this OTU was still higher in LFD + CR mice than in LFD mice ( $1.7 \%$ versus $0.024 \%$, respectively; $P<0.001$, one-way ANOVA). Different from mid-life, the OTUs belonging to Bifidobacterium were higher in LFD + CR mice, but the OTU469 of Desulfovibrionaceae was lower in LFD + CR mice. Some members in the genus Bifidobacterium are well-known probiotic strains ${ }^{25}$, and some in the family Desulfovibrionaceae have previously been found to be positively associated with obesity and inflammation ${ }^{13}$.

The mice had a significantly different gut microbiota structure between the LFD and HFD groups (Supplementary Fig. S3), confirming results of previous studies ${ }^{13,18}$. CR also shifted the gut microbiota in mice fed with high-fat diet but not as dramatic compared with their low-fat diet companions (Fig. 1b,c and Supplementary Fig. S4). In mid-life, 30 phylotypes were selected as key variables for separating the gut microbiota under different food intake conditions (Fig. 2c and 


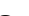

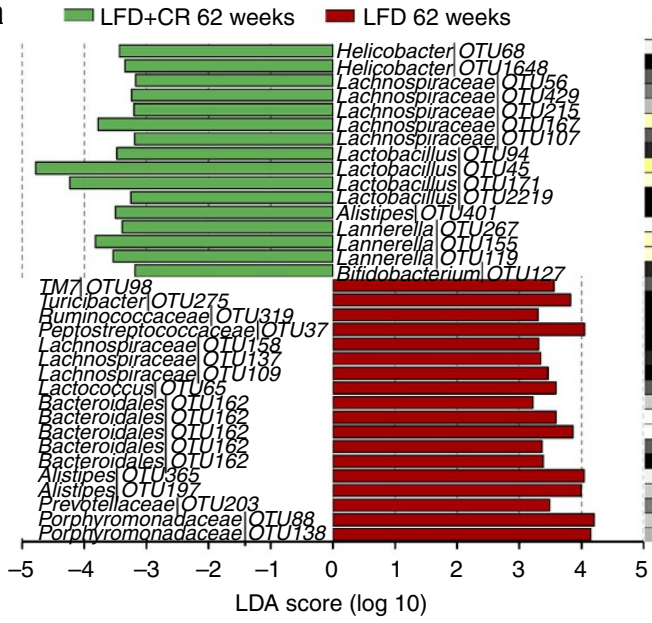

b

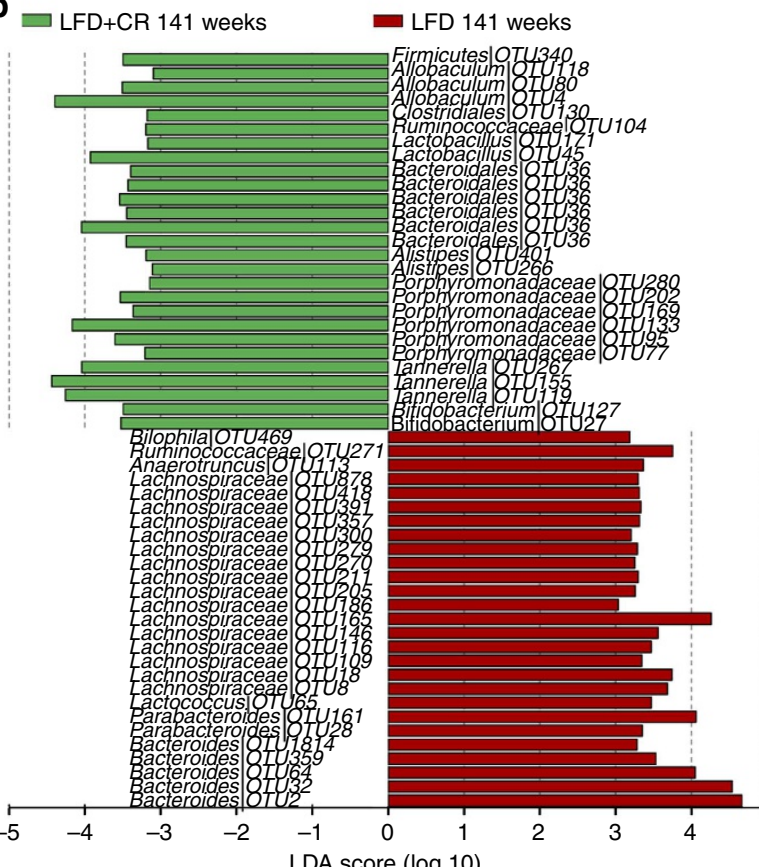

LDA score $(\log 10)$
LFD+CR 62 weeks

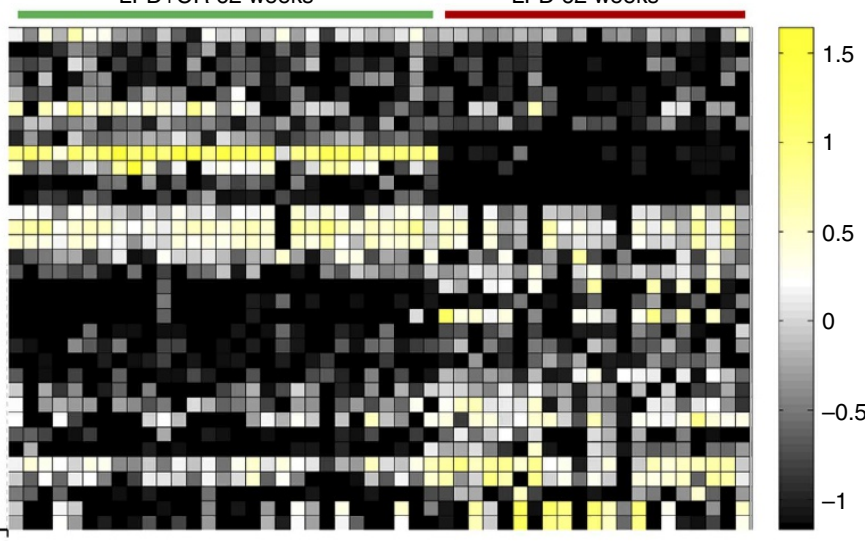

c

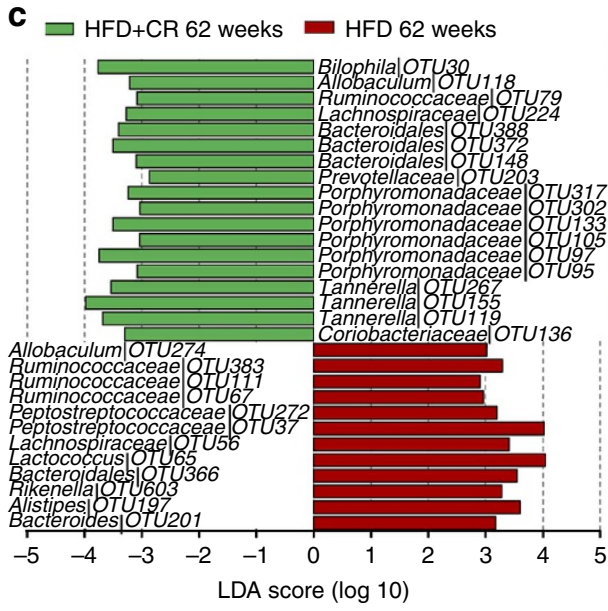

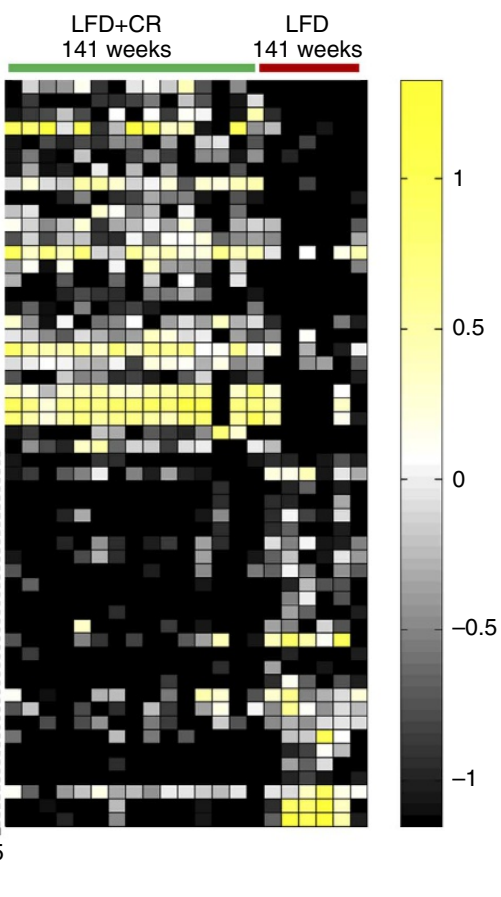

HFD+CR 62 weeks

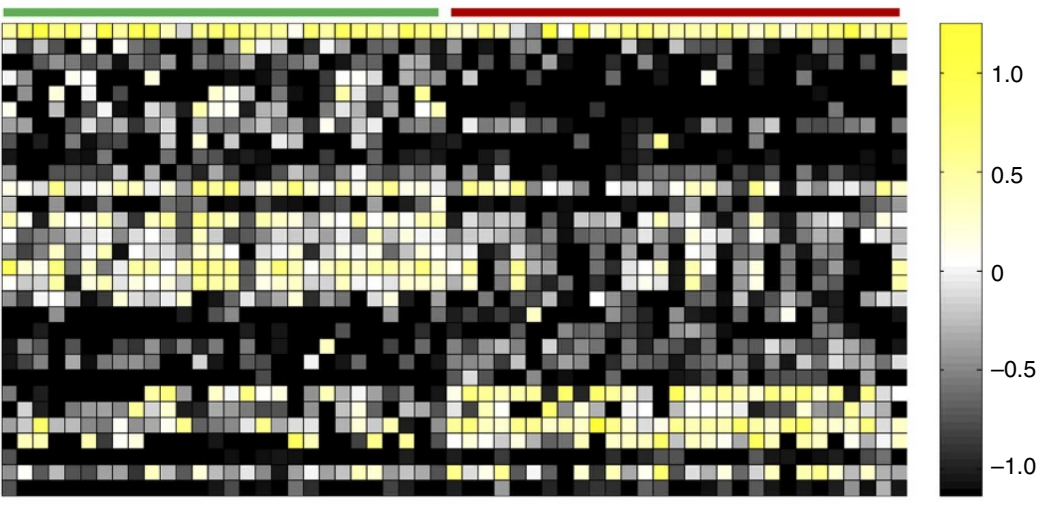

Figure 2 | Key phylotypes of gut microbiota responding to life-long CR identified using LEfSe. (a) LFD $(n=21)$ versus LFD + CR $(n=29)$ mice at 62 weeks. (b) LFD $(n=6)$ versus LFD + CR $(n=15)$ mice at 141 weeks. (c) HFD $(n=28)$ versus HFD + CR $(n=29)$ mice at 62 weeks. The left histogram shows the LDA scores computed for features (on the OTU level) differentially abundant between the $a b$ libitum and CR mice. The right heat map shows the relative abundance (log 10 transformation) of OTUs. 
Supplementary Table S3); 18 of them were higher and 12 were lower in the HFD + CR group than in the HFD group. All the phylotypes in Porphyromonadaceae were higher in the HFD + CR than in the HFD group. Most of the OTUs responding to CR in
HFD + CR mice were not found in LFD + CR mice. Only three OTUs (in Lactococcus (OTU65), Bacteroidales (OTU366) and Peptostreptococcaceae (OTU37), respectively) were reduced, and three OTUs in Tannerella (OTU119, 155 and 267) were increased a

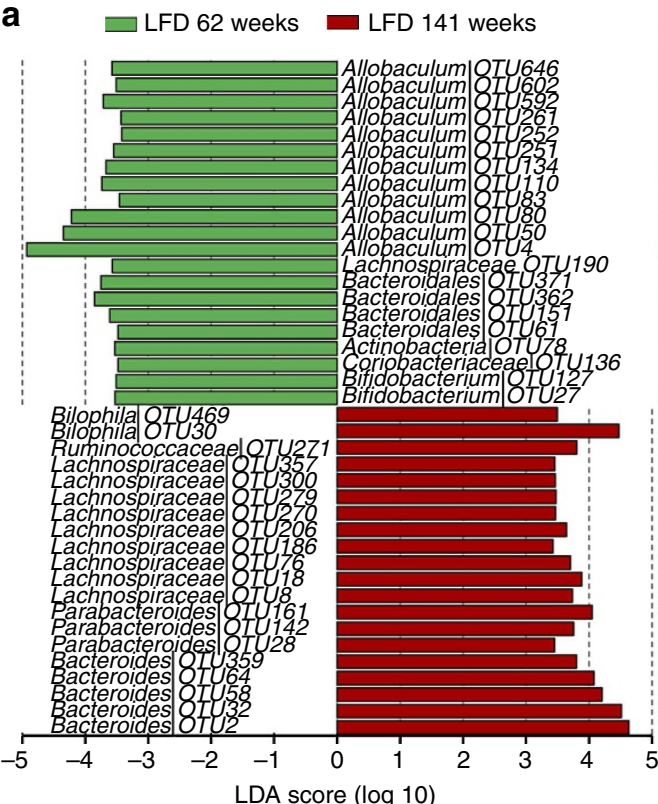

b

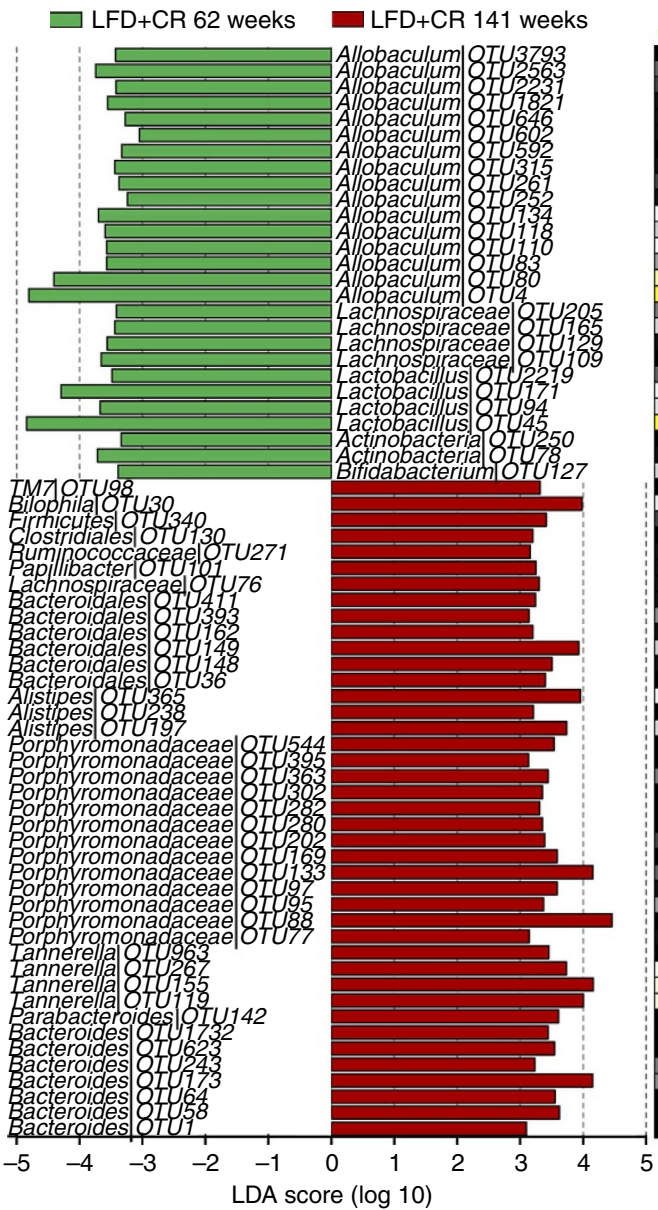

LFD

62 weeks 141 weeks

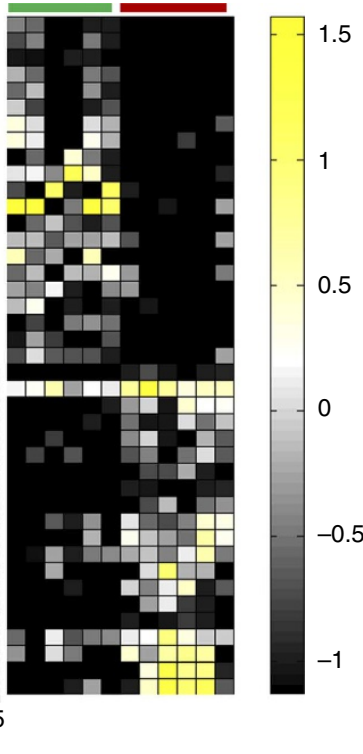

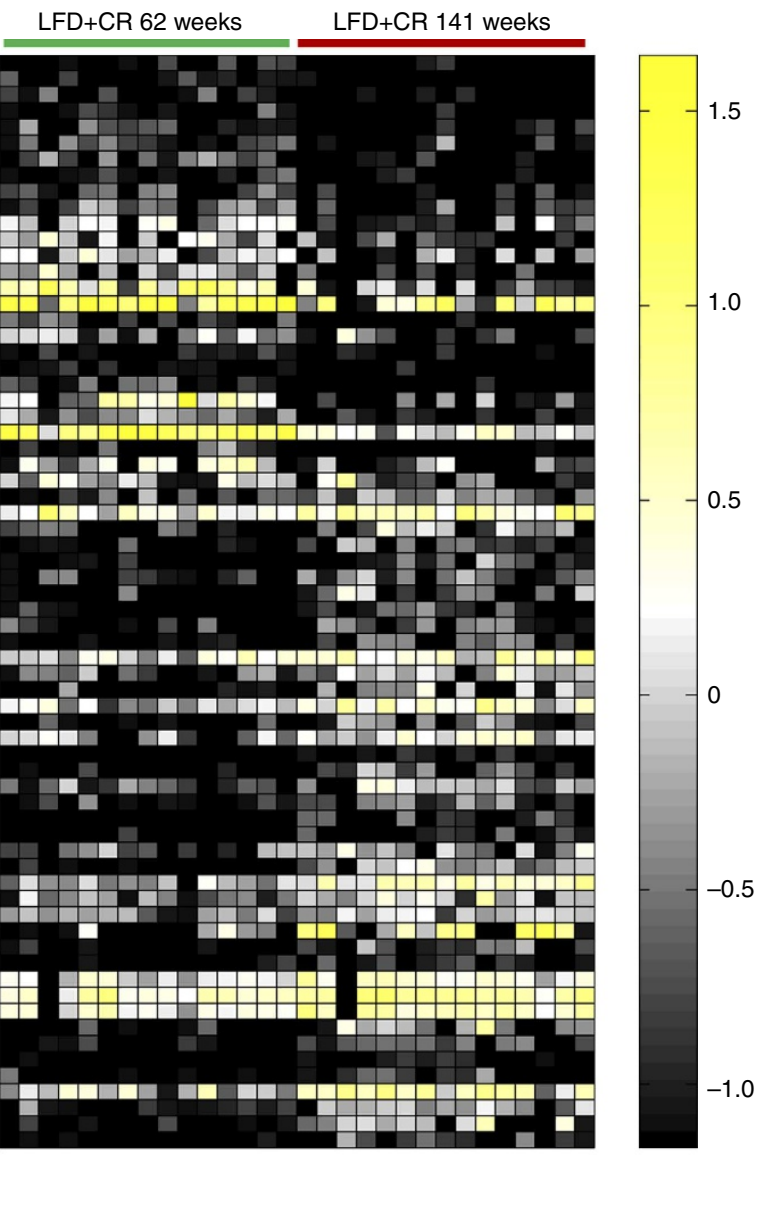

Figure 3 | Key phylotypes of gut microbiota responding to aging. (a) LFD + CR mice $(n=15)$. (b) LFD mice $(n=6)$. The left histogram shows the LDA scores computed for features (OTU level) differentially abundant between 62 and 141 weeks. The right heat map shows the relative abundance (log 10 transformed) of OTUs. 
by CR both with mice fed with high-fat diet and low-fat diet. Because all the HFD mice had died before 141 weeks, we could not obtain any data regarding gut microbiota responding to restriction of high-fat diet intake at the late-life stage of mice.

We also employed partial least square discriminate analysis to confirm the results, and the identified specific phylotypes responding to life-long CR were similar to those from LEfSe (Supplementary Figs S5 and S6).

We also identified a few OTUs that were different between the mice in the exercise group and their ad libitum companions by LEfSe; however, the relative abundance of these OTUs was very low (Supplementary Fig. S7). Conversely, efforts to classify groups with or without voluntary exercise on the same diet with partial least square discriminate analysis did not establish validated models, indicating that the differences between the gut microbiota of animals with or without voluntary exercise were not significant in the present study.

Structural modulation of gut microbiota during aging. Further analysis suggested that CR significantly affected the succession of gut microbiota during aging. Division-level analysis showed that the Firmicutes/Bacteroidetes ratio of gut microbiota in all the mouse groups decreased from 62 to 141 weeks (Fig. 1a). Using LEfSe, we compared the gut microbiota of mice in each group between mid-life and late life to identify the specific phylotypes with OTU levels associated with aging.

In LFD mice, the phylotypes mainly responsible for decrease of the phylum Firmicutes during aging were in the genus Allobaculum (12 OTUs; $28.1 \%$ at 62 weeks versus $0.20 \%$ at 141 weeks) (Fig. 3a and Supplementary Table S4). In LFD + CR mice, not only OTUs in the genus Allobaculum $(29.7 \%$ at 62 weeks versus $7.2 \%$ at 141 weeks) but also OTUs in the genus Lactobacillus $(21.0 \%$ at 62 weeks versus $2.0 \%$ at 141 weeks) made a significant contribution to the decrease of Firmicutes during aging (Fig. 3b and Supplementary Table S5).

Conversely, the increase of the phylum Bacteroidetes during aging in LFD mice was due to the increase of OTUs in the genus Bacteroides (family Bacteroidaceae; $0.84 \%$ at 62 weeks versus $26.7 \%$ at 141 weeks). However, in LFD + CR mice, OTUs in the family Porphyromonadeceae $(9.4 \%$ at 62 weeks versus $28.2 \%$ at 141 weeks), instead of bacteria in Bacteroides, were largely responsible for the increase of Bacteroidetes with age. Thus, the apparent phylum level changes associated with aging were actually mediated by different phylogenetic groups in animals with or without CR treatment.

Correlation of mid-life gut microbiota with lifespan. We next used the Kendall tau rank correlation coefficient to directly measure the correlation between the phylotypes of gut microbiota in mid-life and the lifespan based on two types of diet. We identified that 45 OTUs significantly correlated with lifespan in mice fed on low-fat diet. Except for one Bacteroidales OTU, the remaining 15 OTUs significantly positively correlated with lifespan belonged to Firmicutes. Particularly, eight OTUs in Lactobacillus showed strong correlation with lifespan. The 30 phylotypes negatively correlated with lifespan were distributed in the five Phyla of Bacteroidetes, Firmicutes, Proteobacteria, Actinobacteria and TM7 (Fig. 4a and Supplementary Table S6). In the mice on the high-fat diet, 20 OTUs were positively correlated, and 18 OTUs were negatively correlated with lifespan, most of which were in Firmicutes and Bacteroidetes, except for two Actinobacteria OTUs (Fig. $4 \mathrm{~b}$ and Supplementary Table S7). Because of the strong impact of different diet backgrounds on the gut microbiota, only three OTUs in Lactococcus (OTU65), Bacteroidales (OTU366) and Peptostreptococcaceae (OTU37) showed the
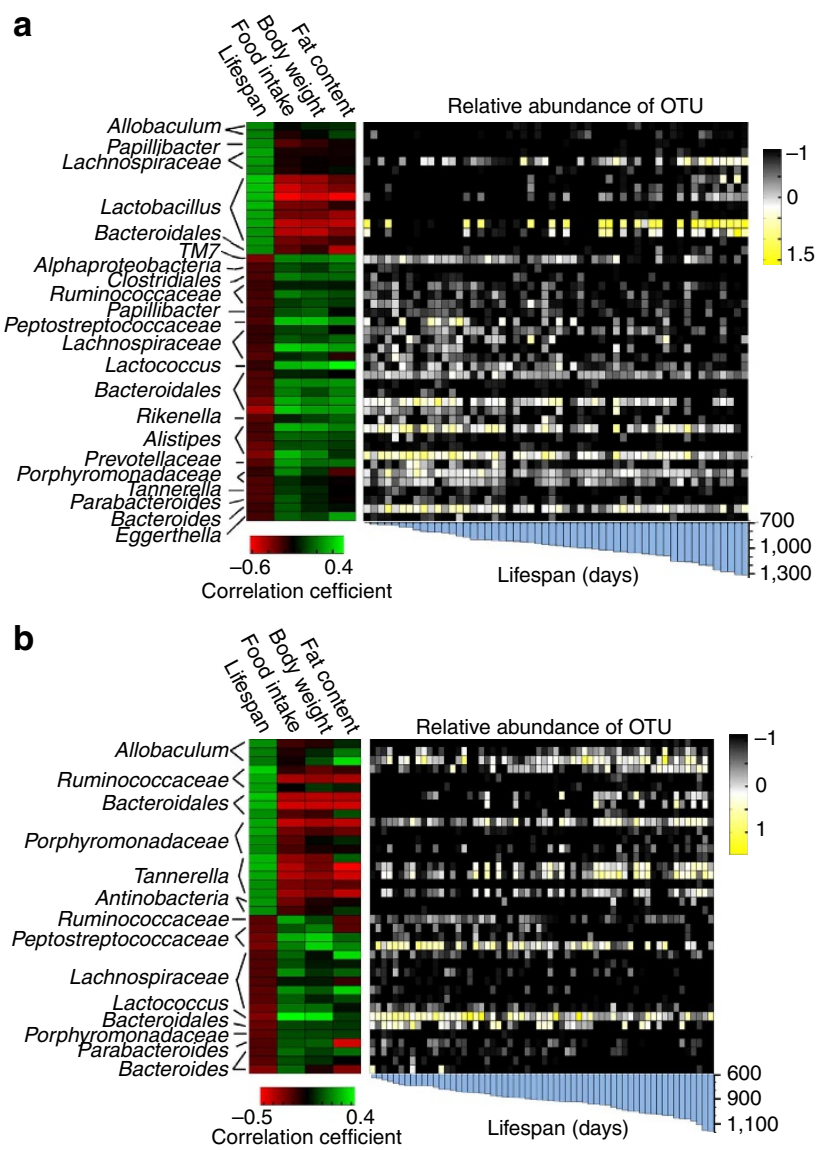

Figure 4 | Correlation of mid-life gut microbiota with lifespan. The phylotypes significantly correlated with lifespan in mid-life (62 weeks) gut microbiota of animals on (a) low-fat diet (LFD, $n=15$; LFD $+C R, n=22$; and LFD + Ex, $n=17$ ) and (b) high-fat diet (HFD, $n=21 ; H F D+C R, n=21$; and HFD $+E x, n=18$ ). The left heat map shows the correlation coefficient between these OTUs and physiological parameters of mid-life. The right heat map shows the relative abundance (log 10 transformed) of OTUs. The bottom bar shows the lifespan of each mouse.

same behaviour both in mice on low-fat diet and high-fat diet. These three OTUs were negatively correlated with lifespan. We also found that the OTUs belonging to the same family or genus could have opposite correlation with lifespan. For example, in the mice fed with low-fat diet, there were three OTUs in Lachnospiraceae positively correlated with lifespan, but the other four OTUs in the same family were negatively correlated with lifespan.

Mid-life metabolic phenotypes, such as food intake, body weight and fat content, were highly correlated with lifespan, a finding that has been reported by Zhou et $a^{22}$. Most of the OTUs significantly positively correlated with lifespan showed strong negative correlation with food intake, body weight and fat content, and vice versa (Fig. 4a,b).

CR reduces antigen load to the hosts from the gut microbiota. Through long-term CR, the relative abundance of the OTUs negatively correlated with lifespan was significantly reduced, and the relative abundance of OTUs positively correlated with lifespan was increased in mice both on low-fat diet and high-fat diet (Fig. 5a,b and Supplementary Figs S8 and S9). The serum levels of lipopolysaccharide (LPS)-binding protein (LBP) from all available animals at mid-life were measured to determine the antigen load from the gut microbiota. Compared with their ab libitum 

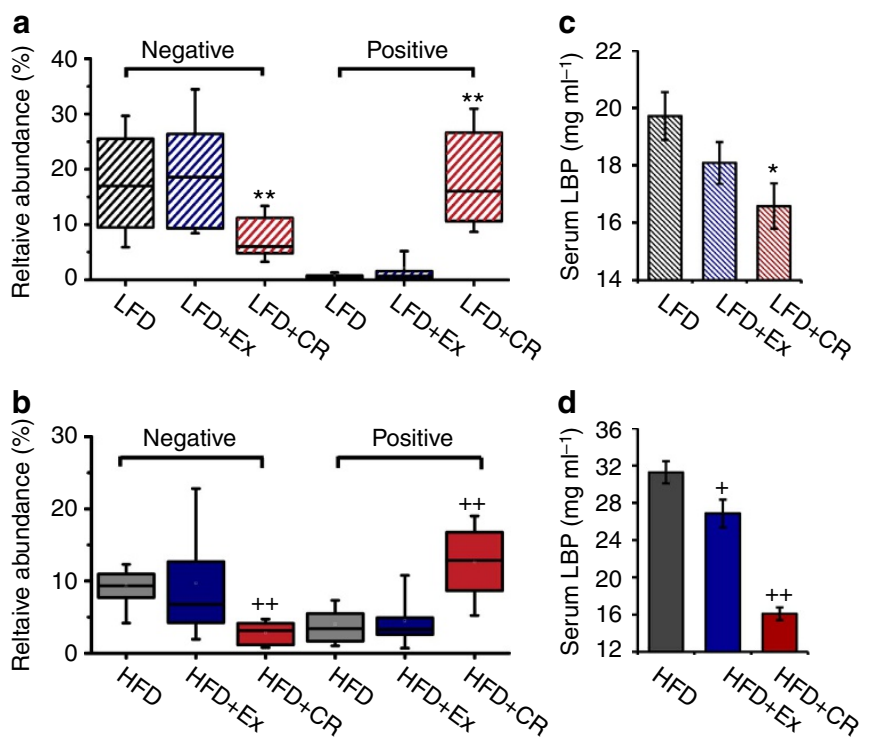

Figure 5 | Gut microbiota-associated antigen load changes. Relative abundance of the phylotypes negatively and positively correlated with lifespan of mice on (a) low-fat diet (LFD, $n=15$; LFD +CR, $n=22$; and $\mathrm{LFD}+\mathrm{Ex}, n=17)$ and (b) high-fat diet $(n=21 ; \mathrm{HFD}+\mathrm{CR}, n=21$; and $\mathrm{HFD}+\mathrm{Ex}, n=18)$ at 62 weeks. The boundary of the box closest to zero indicates the 25th percentile, a line within the box marks the median, and the boundary of the box farthest from zero indicates the 75th percentile. Whiskers (error bars) above and below the box indicate the 90th and 10th percentiles. The serum level of LBP of mice on (c) low-fat diet (LFD, $n=7$; $\mathrm{LFD}+\mathrm{CR}, n=8$; and LFD + Ex, $n=7$ ) and (d) high-fat diet (HFD, $n=8$; $\mathrm{HFD}+\mathrm{CR}, n=8 ; \mathrm{HFD}+\mathrm{Ex}, n=7)$ at 62 weeks (shown as mean \pm s.e.m.). ${ }^{\star} P<0.05$ and ${ }^{\star \star} P<0.01$ versus LFD, $+P<0.05$ and $++P<0.01$ versus HFD by analyses of variance.

companions, LBP levels were lower in mice from the two CR intervention groups (Fig. 5c,d). Conversely, exercise showed no significant impact on both the relative abundance of OTUs correlated with lifespan and the serum level of LBP. These results suggest that modulation of the gut microbiota by CR could significantly reduce the antigen load to the host, contributing to CRinduced lifespan extension.

\section{Discussion}

Among the efforts to understand the mechanisms by which reduced dietary intake improves health and lifespan, the current study is unique in that it focused on the changes of gut microbiota induced by life-long CR to determine whether these changes are associated with improved healthy phenotypes and lifespan.

Different dietary composition can mould the divergent structure of gut microbiota ${ }^{13,26-29}$. For example, human populations with a modern western diet or a rural diet showed distinct gut microbiota structures ${ }^{29}$. The changes in overall structure of gut microbiota induced by high-fat diet versus normal chow may act as an important mediator in the aetiology of obesity and related metabolic diseases via disrupting host lipometabolism regulation ${ }^{15,18,30-32}$ and inducing low-grade inflammation ${ }^{13,14,19,20}$.

In the current study, we also observed shifting of gut microbiota induced by high-fat diet versus low-fat diet (data shown in Supplementary Materials). However, the most interesting result was that, compared with the ad libitum group, mice with $30 \%$ restriction of low-fat diet had a unique gut microbiota, demonstrating that the gut microbiota can be substantially modulated by only restricting the intake of diet. This is different from previous reports that dietary restriction had little effect on the gut microbiota ${ }^{33-35}$. This discrepancy may be due to the short duration of CR, different model systems used (rat and human) and limitation of technology for analysis of gut microbiota used in previous studies. The 454 pyrosequencing method used in the present study, although with its own bias due to the primer design (V3 region targeted) and chosen DNA extraction method, can still allow much deeper and more comprehensive analysis of changes in the gut microbiota responding to $\mathrm{CR}$ than that in previous reports.

Previous studies have suggested that because of the global impact on the physiology of the intestinal tract, including the decrease of intestinal motility, decline in the functionality of the immune system (immunosenescence), and changes in nutritional behaviour and life style of aged people, aging can seriously affect the composition of gut microbiota ${ }^{36-39}$. In the current study, the gut microbiota in all mice changed during aging; however, the most interesting finding was that the shifts of gut microbiota with aging were different among different dietary intervention groups. This finding also suggests that life-long nutritional conditions have an impact on not only the structure and composition of gut microbiota but also the interaction between the host and gut microbiota.

Our approach of directly measuring lifespan as well as measuring dietary and other metabolic conditions, although extremely tedious and costly (more than 150 mice over a time span of 3 years), allowed us to identify the phylotypes of gut microbiota that may be directly associated with lifespan. Correlation analysis between the gut microbiota at mid-life and lifespan identified phylotypes that are positively correlated with lifespan and those that are negatively correlated with lifespan under each of the two dietary backgrounds. On both the high-fat diet and low-fat diet, CR increased those phylotypes that were positively correlated with lifespan, and decreased those that were negatively correlated with lifespan. Phylotypes positively correlated with lifespan may contain beneficial bacteria, whereas those negatively correlated with lifespan may have harmful bacteria such as opportunistic pathogens. For example, the longest-lived and healthiest LFD +CR group had a gut microbiota with astonishingly high populations in Lactobacillus spp. Members in the genus Lactobacillus spp. have been known to inhibit pathogen adhesion to the intestinal wall, protect against pathogen-induced gut barrier disruption and reduce inflammatory cytokines ${ }^{40,41}$. In addition, the LFD + CR group had the lowest level of phylotypes in Streptococcaceae and TM7. It has been shown in humans that some strains in Streptococcaceae can induce mild inflammation, contributing to the disproportionate morbidity associated with chronic wounds among diabetics compared with nondiabetics ${ }^{42,43}$. Members in TM7 may have an important role in the early stages of inflammatory mucosal processes in inflammatory bowel diseases ${ }^{44}$.

The increase of beneficial bacteria such as Lactobacillus and decrease of opportunistic pathogens may reduce antigen load to the host and help alleviate inflammation and metabolic syndrome $13,14,19,20$. Our data showed that CR mice had reduced LBP levels in the serum. LBP is a soluble acute phase protein that binds to LPS, the most abundant gut antigen from the gut with the most potent inflammation-provoking capacity, in eliciting immune responses by presenting LPS to surface patternrecognition receptors, such as CD14 and TLR4, of immune cells $^{45}$. LBP can also bind to antigens produced by Gram-positive bacteria and, thus, may represent one biomarker that links antigen load in the blood and the host inflammatory response ${ }^{46}$. The significant decrease of the ratio of the bacteria negatively and positively correlated with lifespan in animals under CR may minimize antigen entrance into the blood from the gut, a result 
that may constitute a crucial component in CR-mediated benefits to the host ${ }^{13,14}$. Similar to the result of gut microbiota, the measurement of mid-life liver gene expression of mice from the same trial by whole-genome microarrays showed that LFD + CR mice demonstrated a distinctive expression pattern from other groups, which correlated with longevity and health status ${ }^{22}$. Most notably, the gene expression levels of the toll-like receptor signalling pathway and inflammation-related pathways were negatively correlated with lifespan and positively correlated with body weight and metabolic deterioration. Because the tolllike receptor signalling pathway mainly responds to antigens such as endotoxins from the gut microbiota ${ }^{47}$, its downregulation supports the hypothesis that $\mathrm{CR}$ might display reduced antigen load from the gut microbiota to the hosts, possibly by modulating the structure of the gut microbiota to a more balanced state.

The gender of the host is known to have an impact on lifespan, healthy phenotypes and the immune responses ${ }^{48-50}$. In addition, gender may also be involved in the determination of the mammalian gut microbiota. The gender-related bacteria identified from different studies included species of BacteroidesPrevotella, Clostridia, Bacteroidetes and Proteobacteria and so on $^{28,49,51}$. To avoid the influence of gender as a confounding factor, we only used male mice. The response of gut microbiota to $\mathrm{CR}$ in female mice may be different from the results we observed in the current study, and it remains an interesting issue to be investigated.

The molecular cascades between dietary modulation, the gut microbiota and host health remain to be elucidated. The diet can be used by both the host and gut microbiota ${ }^{11,12}$. The competition between the host and gut bacteria for nutrients may determine the composition of the feeding medium for homoeostatic control of microbiota in the lower gut. It is conceivable that under conditions of restricted nutrient availability, as in the case of mice in the LFD + CR group, the host may extract nutrients (such as proteins and fats) more thoroughly, leaving primarily indigestible plant polysaccharides to the colon. In other words, CR without malnutrition might actually increase the relative content of fibre in the animal's diet. This 'oligotrophic condition' with mainly fibre available for gut microbes might promote the growth of beneficial bacteria, such as gut barrier protectors and butyrate producers ${ }^{52}$, but suppress opportunistic pathogens ${ }^{53}$. This hypothesis warrants further studies.

Our results point to the health-promoting potential of a balanced gut microbiota architecture induced by $\mathrm{CR}$, revealing a possible close connection between nutritional modulation of gut microbiota and healthy aging. More mechanistic studies are needed to validate and expand the interesting findings provided here via this microbiome-wide association study ${ }^{54}$. Given the potential key role in mediating the health-promoting actions of $\mathrm{CR}$, an architecturally improved gut microbiota may become a novel surrogate biomarker for the development of effective antiaging dietary interventions.

\footnotetext{
Methods

Animal intervention and samples. The animal experimental procedures, approved by the Institutional Animal Care and Use Committee of the Institute for Nutritional Sciences, CAS, were described previously by Zhou et al 22 . Male C57BL/ $6 \mathrm{~J}$ mice at 5 weeks of age were randomly assigned to one of the six groups ( $n=30$ for each group) and individually caged for a life-long trial: (1) low-fat diet with sedentary activity (LFD), (2) low-fat diet with $30 \%$ CR and sedentary activity $(\mathrm{LFD}+\mathrm{CR}),(3)$ low-fat diet with voluntary running exercise (LFD + Ex), (4) high-fat diet with sedentary activity (HFD), (5) high-fat die with $30 \% \mathrm{CR}$ and sedentary activity (HFD + CR) and (6) high-fat diet with voluntary running exercise (HFD + Ex). All faecal and serum samples for the current study were collected from this animal trial.

Fresh faecal matter was collected from the above animal trial at 62 weeks (LFD, $n=21 ; \mathrm{LFD}+\mathrm{CR}, n=29 ; \mathrm{LFD}+\mathrm{Ex}, n=22$; HFD, $n=28$; HFD $+\mathrm{CR}, n=29$; and
}

HFD + Ex, $n=23$ ), 83 weeks (LFD, $n=16$; LFD + CR, $n=22$; LFD + Ex, $n=19$; HFD, $n=12$; HFD + CR, $n=14$; and HFD + Ex, $n=15$ ) and 141 weeks (LFD, $n=6 ; \mathrm{LFD}+\mathrm{CR}, n=15 ; \mathrm{LFD}+\mathrm{Ex}, n=6 ; \mathrm{HFD}, n=0 ; \mathrm{HFD}+\mathrm{CR}, n=10$; and HFD $+\mathrm{Ex}, n=1)$ of age. At 62 weeks, eight randomly selected mice from each group were humanely euthanized for serum samples collection for LBP analysis. All the faecal samples were stored at $-80^{\circ} \mathrm{C}$ until analysis.

Pyrosequencing of the V3 region of 16S rRNA genes. DNA was extracted using the PSP $\AA$ Spin Stool DNA Plus Kit (Invitek GmbH, Germany). The primers P1 and P2 $\left(5^{\prime}\right.$-NNNNNNCCTACGGGAGGCAGCAG- $3^{\prime}$ and $5^{\prime}$-NNNNNNATTAC CGCGGCTGCT-3') correspond to positions 341 to 534 in the Escherichia coli 16S rRNA gene, with a sample-unique DNA barcode of six-mer sequences at the $5^{\prime}$ end, were used to amplify the V3 region of each faecal sample by PCR. PCR reactions were run in a thermocycler PCR system (PCR Sprint; Thermo electron, Corp., UK) using the following programme: $3 \mathrm{~min}$ of denaturation at $94^{\circ} \mathrm{C}$ followed by 20 cycles of $1 \mathrm{~min}$ at $94^{\circ} \mathrm{C}$ (denaturation), $1 \mathrm{~min}$ for annealing $\left(1^{\circ} \mathrm{C}\right.$ reduced for every two cycles from 65 to $57^{\circ} \mathrm{C}$, followed by one cycle at $56^{\circ} \mathrm{C}$ and one cycle at $55^{\circ} \mathrm{C}$ ) and $1 \mathrm{~min}$ at $72^{\circ} \mathrm{C}$ (elongation), with a final extension at $72^{\circ} \mathrm{C}$ for $6 \mathrm{~min}$. The products from different samples were mixed at equal ratios for pyrosequencing using the GS FLX platform (Roch, Branford, CT, USA).

Bioinformatics and statistical analysis of sequencing data. The standards for quality control of selecting valid reads for analysis were as follows: if a sequence (a) shows no mismatch to the barcode and 16S rRNA gene primer at sequencing end, (b) is more than 100 nucleotides in length, (c) has no more than two undermined bases in the sequence read and (d) finds $>75 \%$ mach to a previously determined $16 \mathrm{~S}$ rRNA gene sequence, as reported previously $y^{5-57}$. The sequences were aligned using NAST, and delineation of OTUs was conducted with DOTUR at $97 \%$ cutoff $^{58}$. The length of the sequence fragments used for the analysis was from 92 to 183 nucleotides (without primer and barcode). The alpha and beta diversities were performed using QIIME ${ }^{59}$. The GAST (Global Alignment for Sequence Taxonomy) process was used to select the top GAST match (es) of the representative sequence of each OTU to assign taxonomic classification ${ }^{60}$. The V3 reference databases (V3 RefDB) and software for GAST analysis were downloaded from http://vamps. mbl.edu/resources/software.php. The V3 RefDB is composed of publicly available, high-quality, full-length $16 \mathrm{~S}$ rRNA sequences from Silva release 92 (http://www. arb-silva.de/) with taxonomic classifications obtained from the RDP Classifier (with a minimum $80 \%$ bootstrap score) and contains $381,203 \mathrm{~V} 3$ tags. The representative sequence of each OTU was assigned the taxonomic classification of the most similar reference sequence or sequences in the V3 RefDB as described previously ${ }^{60}$.

LEfSe $^{24}$ is an algorithm for high-dimensional biomarker discovery and explanation that identifies genomic features (genes, pathways or taxa) characterizing the differences between two or more biological conditions (or classes; see figure below). LEfSe emphasizes both statistical significance and biological relevance, allowing researchers to identify differentially abundant features that are also consistent with biologically meaningful categories (subclasses). We performed LEfSe analysis on the website http://huttenhower. sph.harvard.edu/galaxy. The differential features were identified on the OTU level. The treatment groups or time points were used as the class of subjects (no subclass). LEfSe analysis was performed under the following conditions: (1) the alpha value for the factorial Kruskal-Wallis test among classes is $<0.05$ and (2) the threshold on the logarithmic LDA score for discriminative features is $>2.0$.

Based on two diets, associations between each OTU (filtered for an OTU subject prevalence of at least $10 \%$ ) at 62 weeks and lifespan were determined using the Kendall tau rank correlation coefficient under Matlab (ver. 7.1; The MathWorks, Inc.). The OTU was considered significantly correlated with lifespan for $P<0.05$. Thereafter, the Kendall tau rank correlation coefficient between these selected OTU and physiological parameters (food intake, body weight and fat content) of mid-life was also calculated.

Serum LBP measurements. Blood samples were collected from the tail vein after overnight fasting and centrifuged at 12,000 r.p.m. for $30 \mathrm{~min}$ to pellet blood cells, and the serum was stored at $-80^{\circ} \mathrm{C}$ until further analyses. Serum LBP was determined after a dilution of 1:800 using the Mouse Lipopolysaccharide Binding Protein ELISA Kit (Cell Sciences, Canton, MA, USA) according to the manufacturer's instructions.

\section{References}

1. McCay, C. M., Crowell, M. F. \& Maynard, L. A. The effect of retarded growth upon the length of life span and upon the ultimate body size. J. Nutr. 10, 63-79 (1935).

2. Colman, R. J. et al. Caloric restriction delays disease onset and mortality in rhesus monkeys. Science 325, 201-204 (2009).

3. Fontana, L., Meyer, T. E., Klein, S. \& Holloszy, J. O. Long-term calorie restriction is highly effective in reducing the risk for atherosclerosis in humans Proc. Natl Acad. Sci. USA 101, 6659-6663 (2004).

4. Wolf, G. Calorie restriction increases life span: a molecular mechanism. Nutr Rev. 64, 89-92 (2006). 
5. Guarente, L. Mitochondria-a nexus for aging, calorie restriction, and sirtuins? Cell 132, 171-176 (2008)

6. Wang, Y. et al. Metabonomic investigations of aging and caloric restriction in a life-long dog study. J. Proteome Res. 6, 1846-1854 (2007).

7. Rezzi, S. et al. Metabolic shifts due to long-term caloric restriction revealed in nonhuman primates. Exp. Gerontol. 44, 356-362 (2009).

8. Lederberg, J. Infectious history. Science 288, 287-293 (2000)

9. Qin, J. et al. A human gut microbial gene catalogue established by metagenomic sequencing. Nature 464, 59-65 (2010).

10. Goodman, A. L. \& Gordon, J. I. Our unindicted coconspirators: human metabolism from a microbial perspective. Cell Metab. 12, 111-116 (2010).

11. Sonnenburg, J. L., Angenent, L. T. \& Gordon, J. I. Getting a grip on things: how do communities of bacterial symbionts become established in our intestine? Nat. Immunol. 5, 569-573 (2004).

12. van Hylckama Vlieg, J. E., Veiga, P., Zhang, C., Derrien, M. \& Zhao, L. Impact of microbial transformation of food on health-from fermented foods to fermentation in the gastro-intestinal tract. Curr. Opin. Biotechnol. 22, 211-219 (2011).

13. Zhang, C. et al. Interactions between gut microbiota, host genetics and diet relevant to development of metabolic syndromes in mice. ISME J. 4, 232-241 (2010).

14. Cani, P. D. et al. Metabolic endotoxemia initiates obesity and insulin resistance. Diabetes 56, 1761-1772 (2007).

15. Ley, R. E., Turnbaugh, P. J., Klein, S. \& Gordon, J. I. Microbial ecology: human gut microbes associated with obesity. Nature 444, 1022-1023 (2006).

16. Cani, P. D. et al. Selective increases of bifidobacteria in gut microflora improve high-fat-diet-induced diabetes in mice through a mechanism associated with endotoxaemia. Diabetologia 50, 2374-2383 (2007).

17. Murphy, E. F. et al. Composition and energy harvesting capacity of the gut microbiota: relationship to diet, obesity and time in mouse models. Gut 59, 1635-1642 (2010).

18. Turnbaugh, P. J., Backhed, F., Fulton, L. \& Gordon, J. I. Diet-induced obesity is linked to marked but reversible alterations in the mouse distal gut microbiome. Cell Host Microbe 3, 213-223 (2008).

19. Cani, P. D. et al. Changes in gut microbiota control metabolic endotoxemiainduced inflammation in high-fat diet-induced obesity and diabetes in mice. Diabetes 57, 1470-1481 (2008).

20. Cani, P. D. et al. Changes in gut microbiota control inflammation in obese mice through a mechanism involving GLP-2-driven improvement of gut permeability. Gut 58, 1091-1103 (2009).

21. Na Fei, L. Z. An opportunistic pathogen isolated from the gut of an obese human causes obesity in germfree mice. ISME J. 7, 880-884 (2013).

22. Zhou, B. et al. Midlife gene expressions identify modulators of aging through dietary interventions. Proc. Natl Acad. Sci. USA 109, E1201-E1209 (2012).

23. Knights, D., Costello, E. K. \& Knight, R. Supervised classification of human microbiota. FEMS Microbiol. Rev. 35, 343-359 (2011).

24. Segata, N. et al. Metagenomic biomarker discovery and explanation. Genome Biol. 12, R60 (2011).

25. Veiga, P. et al. Bifidobacterium animalis subsp. lactis fermented milk product reduces inflammation by altering a niche for colitogenic microbes. Proc. Natl Acad. Sci. USA 107, 18132-18137 (2010).

26. Moore, W. E. \& Moore, L. H. Intestinal floras of populations that have a high risk of colon cancer. Appl. Environ. Microbiol. 61, 3202-3207 (1995).

27. Finegold, S. M., Attebery, H. R. \& Sutter, V. L. Effect of diet on human fecal flora: comparison of Japanese and American diets. Am. J. Clin. Nutr. 27, 1456-1469 (1974).

28. Mueller, S. et al. Differences in fecal microbiota in different European study populations in relation to age, gender, and country: a cross-sectional study. Appl. Environ. Microbiol. 72, 1027-1033 (2006).

29. De Filippo, C. et al. Impact of diet in shaping gut microbiota revealed by a comparative study in children from Europe and rural Africa. Proc. Natl Acad. Sci. USA 107, 14691-14696 (2010).

30. Backhed, F. et al. The gut microbiota as an environmental factor that regulates fat storage. Proc. Natl Acad. Sci. USA 101, 15718-15723 (2004).

31. Backhed, F., Manchester, J. K., Semenkovich, C. F. \& Gordon, J. I. Mechanisms underlying the resistance to diet-induced obesity in germ-free mice. Proc. Natl Acad. Sci. USA 104, 979-984 (2007).

32. Ley, R. E. et al. Obesity alters gut microbial ecology. Proc. Natl Acad. Sci. USA 102, 11070-11075 (2005).

33. Mai, V., Colbert, L. H., Perkins, S. N., Schatzkin, A. \& Hursting, S. D. Intestinal microbiota: a potential diet-responsive prevention target in ApcMin mice. Mol. Carcinog. 46, 42-48 (2007).

34. Santacruz, A. et al. Interplay between weight loss and gut microbiota composition in overweight adolescents. Obesity 17, 1906-1915 (2009).

35. Henderson, A. L., Cao, W. W., Wang, R. F., Lu, M. H. \& Cerniglia, C. E. The effect of food restriction on the composition of intestinal microflora in rats. Exp. Gerontol. 33, 239-247 (1998).
36. Kleessen, B., Sykura, B., Zunft, H. J. \& Blaut, M. Effects of inulin and lactose on fecal microflora, microbial activity, and bowel habit in elderly constipated persons. Am. J. Clin. Nutr. 65, 1397-1402 (1997).

37. Ostan, R. et al. Immunosenescence and immunogenetics of human longevity. Neuroimmunomodulation 15, 224-240 (2008).

38. Guigoz, Y., Dore, J. \& Schiffrin, E. J. The inflammatory status of old age can be nurtured from the intestinal environment. Curr. Opin. Clin. Nutr. Metab. Care. 11, 13-20 (2008).

39. Flint, H. J., Duncan, S. H., Scott, K. P. \& Louis, P. Interactions and competition within the microbial community of the human colon: links between diet and health. Environ. Microbiol. 9, 1101-1111 (2007).

40. Zareie, M. et al. Probiotics prevent bacterial translocation and improve intestinal barrier function in rats following chronic psychological stress. Gut 55, 1553-1560 (2006).

41. Bernardeau, M., Guguen, M. \& Vernoux, J. P. Beneficial lactobacilli in food and feed: long-term use, biodiversity and proposals for specific and realistic safety assessments. FEMS Microbiol. Rev. 30, 487-513 (2006).

42. Price, L. B. et al. Community analysis of chronic wound bacteria using $16 \mathrm{~S}$ rRNA gene-based pyrosequencing: impact of diabetes and antibiotics on chronic wound microbiota. PLoS One 4, e6462 (2009).

43. Koyuncu, M., Acuner, I. C. \& Uyar, M. Deep neck infection due to Lactococcus lactis cremoris: a case report. Eur. Arch. Otorhinolaryngol. 262, 719-721 (2005).

44. Kuehbacher, T. et al. Intestinal TM7 bacterial phylogenies in active inflammatory bowel disease. J. Med. Microbiol. 57, 1569-1576 (2008).

45. Sun, L. et al. A marker of endotoxemia is associated with obesity and related metabolic disorders in apparently healthy Chinese. Diabetes Care 33, 1925-1932 (2010).

46. Zweigner, J., Schumann, R. R. \& Weber, J. R. The role of lipopolysaccharidebinding protein in modulating the innate immune response. Microbes Infect. 8 , 946-952 (2006).

47. Kawai, T. \& Akira, S. The role of pattern-recognition receptors in innate immunity: update on Toll-like receptors. Nat. Immunol. 11, 373-384 (2010).

48. Barrett, E. L. \& Richardson, D. S. Sex differences in telomeres and lifespan. Aging Cell 10, 913-921 (2011).

49. Markle, J. G. et al. Sex differences in the gut microbiome drive hormonedependent regulation of autoimmunity. Science 339, 1084-1088 (2013).

50. Morell, V. Zeroing in on how hormones affect the immune system. Science 269, 773-775 (1995).

51. Li, M. et al. Symbiotic gut microbes modulate human metabolic phenotypes. Proc. Natl Acad. Sci. USA 105, 2117-2122 (2008).

52. Gibson, G. R. Dietary modulation of the human gut microflora using the prebiotics oligofructose and inulin. J. Nutr. 129, 1438s-1441s (1999).

53. Naughton, P. J., Mikkelsen, L. L. \& Jensen, B. B. Effects of nondigestible oligosaccharides on Salmonella enterica serovar typhimurium and nonpathogenic Escherichia coli in the pig small intestine in vitro. Appl. Environ. Microb. 67, 3391-3395 (2001).

54. Wang, T. et al. Structural segregation of gut microbiota between colorectal cancer patients and healthy volunteers. ISME J. 6, 320-329 (2012).

55. Margulies, M. et al. Genome sequencing in microfabricated high-density picolitre reactors. Nature 437, 376-380 (2005).

56. McKenna, P. et al. The macaque gut microbiome in health, lentiviral infection, and chronic enterocolitis. PLoS Pathog. 4, e20 (2008).

57. Sogin, M. L. et al. Microbial diversity in the deep sea and the underexplored "rare biosphere". Proc. Natl Acad. Sci. USA 103, 12115-12120 (2006).

58. Zhang, C. et al. Structural resilience of the gut microbiota in adult mice under high-fat dietary perturbations. ISME J. 6, 1848-1857 (2012).

59. Caporaso, J. G. et al. QIIME allows analysis of high-throughput community sequencing data. Nat. Methods 7, 335-336 (2010).

60. Huse, S. M. et al. Exploring microbial diversity and taxonomy using SSU rRNA hypervariable tag sequencing. PLoS Genet. 4, e1000255 (2008).

\section{Acknowledgements}

We thank C.B. Newgard (Duke University) for the initial design of the animal intervention study. This work was supported by the following grants: China MOST (2012CB524900, 2011CB910900, 2007DFC30450, 2009ZX10004-601, 2006BAI11B08, 2088AA02Z315, and 2007CB513002); NSFC (No. 30730005, 3112106, No. 30988002 31230036, 81021002 and 91213306); CAS (No. KSCX2-EW-R-09), Shanghai STC (No. 10XD1406400), and US ADA Research Award 7-06-RA-165.

\section{Author contributions}

L.Z., Y.L. and Y.Z. designed and supervised the research project and wrote the paper. S.L., L.Y., P.H. and W.L. performed the animal studies and analysed the physiological data. C.Z. collected faecal samples, designed pyrosequencing barcodes, conducted pyrosequencing and bioinformatics analysis and wrote the paper. S.W. and G.Z. did the pyrosequencing. M.Z. and X.P. helped analyse the data. 


\section{Additional information}

Access code: Sequence information: all sequence data have been deposited in the NCBI Sequence Read Archive under accession code SRA012394.1.

Supplementary information accompanies this paper at http://www.nature.com/ naturecommunications

Competing financial interests: The authors declare no competing financial interests.
Reprints and permission information is available online at http://npg.nature.com/ reprintsandpermissions/

How to cite this article: Zhang, C. et al. Structural modulation of gut microbiota in lifelong calorie-restricted mice. Nat. Commun. 4:2163 doi: 10.1038/ncomms3163 (2013).

(c) (1) $\Theta$ This work is licensed under a Creative Commons Attribution(cc) No $\bigoplus_{\mathrm{BC}}$ NonCommercial-NoDerivs 3.0 Unported License. To view a copy of this license, visit http://creativecommons.org/licenses/by-nc-nd/3.0/ 